generations of sib mating in 10 lines of Drosophila melanogaster, the character being the total number of abdominal bristles on two sternites. This character, from studies by myself and others is considered to be under mainly additive genetic control with very little true environmental variance, so that there should be no inbreeding depression nor much fluctuation from generation to generation. Nevertheless all 10 lines drop sharply in mean at the start of inbreeding, and most of them show striking fluctuations over generations 15-20, when inbreeding should have eliminated almost all the genetic variation within each line. This does not fit in with expectation, and I hope that someone may be incited to repeat the experiment.

ERIC REEVE

Institute of Animal Genetics, University of Edinburgh

\section{Henderson's Dictionary of Biological Terms. 10th Edition. Edited by Eleanor LaWRENCE. Harlow, Essex: Longman Group UK, 1989. 637 pages. Hard cover £17.95. ISBN 0582463629 .}

This book will be of considerable value to any biologist who ventures outside his own specialist field(s) - which probably means all of us. Revision of the previous ninth edition of 1979 was concentrated on bringing the dictionary up to date in the most rapidly advancing areas of biology, particularly biochemistry, cell biology, genetics and immunology. The 18000 or so definitions, or at least those I have read, are informative and appear to be accurate, and they are quite extensive. To take a few examples from random pages without giving the actual definitions, one finds allometry (without the logarithmic basis of its approach), cribellum = cribrellum, critical group (in taxonomy), demilunes, disassortative mating, $E$ face, numerous words beginning with eco- or ect-, $F$ factor and F-primes in Escherichia coli, homeobox or homoeobox, exons and introns, kappa particles; and moving on a few hundred pages we meet Sewall Wright/effect (defined under genetic drift) but not Sewall Wright's path coefficients, the Shine-Dalgarno sequence, shotgun cloning, $P$ face, $P$ factors, reporter gene, lac operon, sphenosquamosal, and so on. $k$-selected and $r$-selected species are defined; $T i$ and $R i$ plasmids of Agrobacterium species are explained along with their value in genetic engineering.

Gene symbols and most medical terms are sensibly omitted, but the more common human genetic diseases are briefly described. There is a particularly helpful listing of abbreviations and acronyms at the beginning of items under each letter of the alphabet, many of them necessary to understand recent research in immunology, biochemistry and cell biology. The book begins with its own list of abbreviations, units and conversion factors, SI prefixes, the Greek alphabet and common Greek and Latin noun endings; and it ends with 7 appendices giving selected chemical structural formulae, outlines of an up-to-date classification of living forms, and a list of the common chemical elements with their symbols, atomic numbers and atomic masses.

I am not clear whether Eleanor Lawrence is the editor or the main or sole author, or both, since no other names are mentioned. In any case she is warmly to be congratulated on her efficient handling of the 18000 terms and symbols and the very complete biological coverage achieved. I think this book will not only be very useful to all who get access to it, but it will also beguile many of them as it has beguiled me with the numerous terms they have never heard of. At the very reasonable price of just under $£ 18.00$, for a well-bound hard-cover book of such size, it should find its way into every biological library and onto many additional shelves. The diligent reader will come across quite a number of terms which the inventors might well have done without, though they are no doubt proud of them. I will mention just one, 'osmatic', meaning to have a sense of smell; or should we also dispose of 'to oscitate' for to yawn or gape?

ERIC REEVE

Institute of Animal Genetics, University of Edinburgh 